\title{
The Role of Affects in Culture-Based Interventions: Implications for Practice
}

\author{
Terri Mannarini*, Enrico Ciavolino, Mariangela Nitti, Sergio Salvatore \\ Department of History, Society and Human Studies, University of Salento, Lecce, Italy \\ Email: "terri.mannarini@unisalento.it \\ Received May $5^{\text {th }}, 2012$; revised June $7^{\text {th }}, 2012$; accepted July $10^{\text {th }}, 2012$
}

\begin{abstract}
The study aimed to show the relevance of two types of sense-making processes (i.e. cognitive and affective) in culture-based interventions. A hierarchical model based on a psychodynamic theoretical framework was tested. According to this model, a generalized affective meaning connoting the whole field of participants' experience would have a regulative, downward, and causal influence on the specific meanings related to the issues addressed by the intervention. Secondary analyses - namely PLS Path Modeling with higher order constructs - were performed on a dataset resulting from a survey involving three hundred and ninety freshmen enrolled in a psychology course at the University of Salento, Italy. These analyses were aimed at detecting the anticipatory images of the University. Our findings provide evidence supporting the theoretical model proposed. Implications for culture-based interventions are discussed.
\end{abstract}

Keywords: Cultural-Based Intervention; Affective Semiosis; PLS Path Modeling

\section{Introduction}

In the development of a theoretical framework for psychosocial interventions, and the implementation of such interventions, many psychologists have mainly based their proposals on the ecological perspective, which was developed in previous decades by scholars such as Kelly $(1966,1987,2006)$. This ecological approach has enabled psychologists to address major concerns of the field, such as detecting how social systems influence the life of individuals and communities, and how to change these systems so as to increase people's well-being (Hirsch, Levine, \& Miller, 2007). In striving to overcome the limitations of individual-level theorizing, the ecological model adopts a systemic view, emphasizing the relevance of linkages and interactions among the parts of the system, as well as its dynamic and constantly changing nature. In addressing the individual-environment relationship, a basic tenet of the ecological perspective is that "the theory driving the intervention is about the dynamics of the context or system, not the psyche or attributes of the individuals within it" (Hawe, Shiell, \& Riley, 2009: p. 269).

Within the ecological paradigm, multilevel interventions have recently gained momentum, as illustrated by the 2009 special issue of the American Journal of Community Psychology, edited by Jean J. Schensul and Edison Trickett. This multilevel concept can be traced back to Bronfenbrenner (1979), who identified four interconnected systems that frame all human transactions and influence human behavior: the microsystem, mesosystem, exosystem, and the macrosystem, which together make up the ecosystem. Each of these systems affects a variety of aspects of individual and community life, thereby contributing to the well-being and disease of individuals and groups. From this perspective, the rationale behind multilevel interventions is that changes need to be made both at the level

*Corresponding author. of individuals, and of the social context in which they reside. Nevertheless, among interventions couched in the ecological perspective, there is still considerable variation in empirical results achieved. This variation can be parsed by identifying two broad theoretical categories of interventions: those emphasizing the impact of changes in the context surrounding the individual (environment-based), and those emphasizing the relevance of sociocultural processes in changing systems (culture-based).

An example of environment-based interventions is STEP (the School Transitional Environment Project) (Felner \& Adam, 1988; Felner, Favazza, Shim, Brand, Gu, \& Noonan, 2001). STEP seeks to facilitate the transition from elementary to junior high school by modifying specific elements of the school context. More specifically, STEP seeks to accomplish the following goals: 1) create smaller learning environments and provide a stable set of peers to increase the student's sense of connectedness, thereby reducing the degree of complexity that the student entering junior high confronts; and 2) restructure the roles of homeroom teachers so that they provide greater support for entering students. Findings from STEP showed that modifications in the school context helped students to cope with transitional requirements. Nevertheless, it was acknowledged that such changes were "necessary, but certainly not sufficient, elements to obtain the gains in achievement and performance that were above those levels at which the student entered" (Felner et al., 2001: p. 189).

Interventions in the culture-based category are sensitive to the cultural nature of context and emphasize the need for obtaining local knowledge and community involvement in the whole process of the intervention (i.e., development, implementation, and analysis) (Schensul, 2005), according to a collaborative and participatory pattern. Interventions of this category aim to change the system under scrutiny by mobilizing internal resources. Local knowledge, which can be defined as the local culture of individuals and groups, plays a central role 
among these internal resources. Following Trickett (2009), the concept of (local) culture can be defined as "the broad cultural history of community groups as reflected in local contexts that specify how that history is expressed in local settings, relationships with cultural outsiders, and the definition of community issues to be solved" (p. 259). Hence, local culture can be regarded as the outcome and the process of sense-making by which people come to be able to think or communicate about their experience and assign it meaning, value, and relationships to other events. Culture-based interventions compensate for the lack of emphasis put on the role of extra-subjective factors by increasing interventionists' awareness of the sense-making processes that mediate the relationship between the individuals and the context (either physical, social, and symbolic) they are embedded in, or, alternately phrased, the local dynamics of meaning construction.

In the current paper, the authors argue that culture-based interventions have paid much more attention to the cognitive, rather than the affective, processes of sense-making. This is true even within the socio-cultural as well as within the dialogical vision of sense-making (Salgado \& Clegg, 2011; Valsiner \& Rosa, 2007). Further, the authors argue that the lack of recognition of the role of affects in sense-making has led researchers and practitioners to focus exclusively on the meanings related to the specific objects ${ }^{1}$ addressed from time to time by single interventions, and this has shaped the resulting interventions accordingly. However, from a psychodynamic perspective, there is evidence that sense-making also entails the construction of generalized meanings that, though distinct from the specific objects about which individuals are stimulated to verbalize, drive and rule the way the same individuals perceive and represent such objects (Salvatore \& Freda, 2011; Cabell \& Valsiner, in press). The aim of the current paper is to test a model based on a psychodynamic theoretical framework that shows the relevance of such generalized meanings and their relationship to object-specific meanings. Firstly, readers are provided with an overview of the theoretical framework underlying the model and then with the discussion of relevant pieces of evidence. Next, a statistical formalization of the model is illustrated. Finally, implications for culture-based interventions are highlighted.

\section{The Theoretical Framework: Affect and Sense-Making}

As stated above, the concept of local culture was defined as the process and the outcome of sense-making. Signs are mobilized by people as they engage in activities that require intersubjective engagement. Hence, sense-making is intrinsically dialogical. It is argued that a core component of sense-making is the affective nature of our experience. Authors have extensively elaborated on this dynamic-referred to as affective semiosis-elsewhere (Salvatore \& Freda, 2011; Salvatore \& Zittoun, 2010), so only the basic tenets will be recapitulated here.

In this paper, the notion of affect is defined in psychodynamic and semiotic terms (Salvatore \& Zittoun, 2011) - that is, as a process of connotation of the object that motivates a specific disposition to act. According to psychoanalytic theory (Freud, 1933[1932], 1964; see also Bucci, 1997; Matte Blanco, 1975), the affective connotation is the product of a kind of thinking characterized by the predominance of the primary process. The primary process is the way the mind's functioning orients toward homogenizing and generalizing the objects of experience. Insofar as the primary process is predominant in a person's way of thinking, that person does not experience the discrete objects in their singularity and semantic specificity; rather, the objects are interpreted as the generalized class they belong to, as merged with the whole they are part of.

Psychoanalytic theory does not consider the primary process as being opposed to the secondary process (the secondary process is logical, rational thinking, which is centered on the identification of the differences among the elements and constrained by their semantic specificity). Rather, primary and secondary processes are seen as complementary. The secondary process works in terms of setting categorical distinctionsrelationships within the homogenizing way of functioning of the primary process. Thus, the primary and the secondary processes are two coextensive mental functions: every psychological process is the output emerging from their interaction, and therefore, thinking is always a mixture of both.

The psychodynamic idea that basic meanings emerge from the encounter between conscious and unconscious dynamics provides a meaningful way of modeling affects. On this basis, it was proposed to define affects as hypergeneralized and homogenized meanings. Generalization is the process through which individuals and groups recognize an object, not for its distinctiveness and uniqueness, but as correspondent to the whole class of objects that are associable with it-i.e. the motherness rather than the mother. Examples of generalization are numerous: consider the awe one can feel in front of a superior or another figure with some authority (a teacher, a policemen, an elderly relative etc.). Such a feeling cannot be related to the actual attitude and power of the figure; rather, these emotions reflect a meaning that individuals attribute to the generalized class of the authoritative and powerful figures they identify their interlocutor with, rather than to the specific person. Interwoven with generalization is homogenization; if every object of the class is the class itself, all the qualities and characteristics of the class are attributed to the single object. As a consequence, every object is made identical-homogenized - to every other object that belongs to the same class. Stereotypic thought evidently shows hints of the affective mechanism discussed. All the objects that are projected in the stereotypical class are confused with each other, regardless of their individual specificity, and treated according to all the properties associated with the class.

As a consequence of (hyper)generalization and homogenization, the reference of the affective semiosis is never a discrete object $^{1}$ (i.e. a person, an event, a thing), but the relationship between the subject and the object. More precisely, the content is not even the relationship as a discrete "thing"; rather, it is the whole subjective field of experience associated with the practice of the situated encounter with the world. One can pick up a hint of this level of experience when a new situation is encountered. In those circumstances, sometimes one can experience the situation as something endowed of global value (as threatening, warm, distancing...), before differentiating the experience through a specific attentive focus. Such an affective ex-

\footnotetext{
${ }^{1}$ Here and henceforth the term "object" is used in broad sense, for denoting any kind of discrete pattern of experience- - e.g. a person, a things, an event, as well as an institution, an element of the social or physical environment, and so forth.
} 
perience is the subjective (or rather intersubjective) construction of the environment. Hence, a specifically psychological connotation to the notion of context is assumed-as the affective hypergeneralized meaning in terms of which people connote the field of experience.

\section{Evidence of the Salience of Affective Semiosis}

Both inside and outside the psychodynamic field, there is empirical evidence supporting the idea of the salience of a dimension of generalized meaning. From a social psychology perspective, studies on the semantic differential (Osgood, Suci, \& Tannenbaum, 1957) have highlighted that when subjects are asked to evaluate different objects they systematically connote such objects in a latently similar way, regardless of the semantic difference among them. In short, different objects elicit a similar generalized meaning. Moreover, in spite of the fact that this literature has developed essentially outside the psychodynamic field, the authors have recognized the affective nature of this process of meaning construction, due to its generalized, basic, elementary as well as conative nature. These findings offer evidence that the mind carries out sense-making that is the expression of affective generalized classes of meanings in oppositional relationships (good versus bad; full-of-power vs empty-of-power; active vs passive) and is referred to the global field of the experience as a whole. At this level of semiosis, objects are interpreted as part of such an affective field (for instance, as merged with the context as good and powerful), regardless of their semantic specificity. Further evidence that affective semiosis works independently of the cognitive computation focused on the semantic content is provided by the classical studies of Turvey, Fertig and Kravetz (1969), which showed that the priming effect works also when the prime and the target stimuli have no semantic relationship but share the same affective meaning - as it is identified by their similar position on the latent dimensions depicted by the semantic differential (e.g. two objects both connoted as fine and powerful). Similarly, based on a priming procedure, Murphy and Zajonc (1993) showed that preferences do not require semantic elaboration of the stimulus; this leads to the conclusion that the affective elaboration of the experience comes before and guides the semantic-cognitive computation.

A different stream of studies provides convergent evidence. Several surveys aimed at mapping the people's way of connoting specific social objects (e.g. Carli \& Salvatore, 2001; Guidi \& Salvatore, in press; Salvatore, Mannarini, \& Rubino, 2004), have systematically found the same pattern of representation, whatever the object under scrutiny (i.e. the psychologist, the school attended, the University, the community health services) and whatever the socio-cultural context of the study (e.g. urban vs rural areas). In all these studies, the representation of the target object results from the respondents' global image of the context. For instance, if one has a negative vision of the context, she connotes the psychologist as a cheat, assimilating him with a self-styled magician, or represents the teacher of her own school as incompetent and untrustworthy; on the contrary, if one has a positive image of the context, then this connotation reverberates on the psychologist, the teacher, the community health system and any other discrete object that is part of the field so connoted, which thus is represented as trustable, skillful, efficient and the like.

In a recent study aimed at surveying the customer satisfac- tion of a national sample of parents in the Italian school system (Salvatore, Mossi, \& Cazzetta, 2007), two findings were reported that are worth mentioning here: First, the rate of overall satisfaction was uncorrelated with the analytic judgments concerning the specific issues involved in the school service. This finding was seen as an indication that the overall satisfaction with the service was the result of a synthetic global evaluation of the experience of relating with the school, instead of a reflection of a functional examination of the elements and aspects (content, process, performance modality, etc.) underlying the service. Second, the analytic judgments turn out to be strongly associated with the corresponding level of relevance these same issues have to the school service. This was taken as an indication that the attribution of relevance did not depend on a functional (hence, semantic-based) analysis of the value of the issue, but rather on the respondent's liking for the issue; the more they liked it (the more they were satisfied with it), the more they felt it to be important. Translating these findings into the terms of our discussion, they highlight the fact that in an evaluative task - at least when there is a high degree of involvement of the subjective sphere (that is, as a level of satisfaction) - people tend to make generalized, affective categorizations without the constraint of being anchored to the functional analysis of the semantic content.

In sum, the findings mentioned above show the salience of a level of affective sense-making:

1) Working in a generalized and homogenizing way, not constrained by the semantic differentiation of the objects;

2) Referring to the whole field of experience rather than to discrete objects, the latter being connoted by reason of their embeddedness in the field (i.e., an object is good or bad because it is part of a field connoted as good or bad);

3) Playing a super-ordered, regulative role in the semantic elaboration of discrete elements of the field of experience (i.e., the affective connotation of the field of experience feeds the way of thinking about the specific objects, and therefore the commitment and attitude toward them).

\section{A Model of Affective Sense-Making}

As seen above, a plurality of sources of evidence (along with many clinical observations) highlights the presence of affective sense-making, as well as its effect on how people think and act. Yet, this literature has not yet provided an analytic model of the relationship between the affective sense-making of the field of experience (henceforth: affective meaning) and the cognitive processes of semantic elaboration of the discrete objects of the field (henceforth: semantic meaning). This absence constrains the chances of empirical validation of the theory, therefore reducing the possibility of its development and integration with other theories of sense-making and social action. Above all, it prevents the possibility of developing strategies and methodologies of psychosocial intervention that take into account the interlacement between affective and semantic meaning.

The current study takes step toward addressing this issue. A formal quantitative model of the relation between the affective and semantic meaning was proposed, and this model was given its first empirical test. The model is hierarchical: it encompasses first order factors (i.e. semantic meanings concerning discrete objects) and second order factors (i.e. affective meaning concerning the whole field of experience).

$$
\mathrm{SM}=\mathrm{f}\left(\mathrm{A}_{\mathrm{fe}}, \mathrm{S}_{\mathrm{do}}\right)
$$




$$
\mathrm{S}_{\mathrm{do}}=\mathrm{h}\left(\mathrm{A}_{\mathrm{fe}}\right)
$$

where:

$\mathrm{SM}=$ Sense-making;

Afe $=$ Affective meaning of the field of experience;

Sdo $=$ Semantic meaning of discrete objects.

In brief, the formula represents in a formalized language the idea that the sense-making process depends on the relationship between affective categorization and cognitive computation, where affective categorization comes first.

\section{Aim and Hypothesis of the Study}

The study aimed to test the hierarchical structure of the model, highlighting the super-ordinate role played by the affective meaning. According to the model of sense-making proposed above, it was hypothesized that:

a) Sense-making has two dimensions of functioning: semantic and affective meaning;

a1) Semantic meaning concerns the representation of discrete objects;

a2) Affective meaning concerns the totality of the field of the experience;

b) Affective meaning connotes the field of experience in terms of generalized and homogenized classes of meanings;

c) Affective meaning is super-ordinate to semantic meaning - namely, it has a regulative, downward, causal influence on semantic meaning.

\section{Method}

To test the model, a secondary analysis on a dataset resulting from a survey involving freshmen in the undergraduate psychology program at the University of Salento, Lecce, Italy (Venuleo, Mossi, \& Salvatore, submitted) was performed. The survey was aimed at detecting anticipatory images of the university expressed by freshmen at the moment of their first contact with the new educational context. Two specific hypotheses about such anticipatory images were assumed: 1) they would impact the middle and long-term academic performance of students; 2) they would constitute a valuable knowledge base for the elaboration of effective educational policies.

\section{Sample}

A non-representative sample of the student population made up of three hundred and ninety freshmen intending to join the undergraduate psychology program at University of Salento were involved in the survey. All cases with missing values were excluded from the analysis. The resulting reduced sample was composed of $N=366$ cases, of which 312 were female $(85.25 \%)$. The students' age ranged from 18 to 50 ; most of them $(84.43 \%)$ were 18 to $25,7.65 \%$ from 26 to 35 , and about $6 \%$ were older than 36 . More than half the sample $(59.56 \%)$ was unemployed, $12.3 \%$ had a full-time jobs, and $23.77 \%$ had an odd or part-time jobs (4.4\% did not provide this information).

\section{Procedures}

Freshmen were administered a paper-and-pencil questionnaire in September 2009, as freshmen were summoned to take the admission test for the undergraduate psychology program. Before completing the test, students were given 90 minutes to fill in the questionnaire about anticipatory images of the uni- versity. Data entry was carried out with the support of an optic reader. Data analyses were performed using MATLAB software, Version R2009b.

\section{Instruments}

The original questionnaire, composed of 135 items, was designed to map the students' semantic meaning (opinions, judgments, evaluations) of four objects: 1) $\xi_{1}$, Commitment to the university (motivations, expectations); 2) $\xi_{2}$, Trustworthiness of the social system (reliability of social structures, e.g., local administration and services); 3) $\xi_{3}$, Psychology as a profession (functions of psychologists and psychological knowledge); and 4) $\xi_{4}$, General values (morality, respect of rules, etc.). All variables were measured with a 4-point Likert-like scale, either in the format of agreement (from "fully disagree" to "fully agree") or intensity (from "not at all" to "very much"). For the current study, a subset of 19 items was selected, also called manifest variables (see Table 1), based upon the results of an item analysis performed on the whole dataset. Items were selected according to the strength of the contribution they provided to the measurement of the semantic meaning attributed to the four objects upon which the questionnaire was focused.

\section{Data Analysis. PLS Path Modeling for Higher-Order Constructs}

To test the hypothesized model, a procedure of analysis based on PLS Path Modeling with high order constructs was implemented. Due to its ability to estimate complex models, PLS Path Modeling can be used to investigate models at a high level of abstraction. The basic PLS design was first performed in 1966 by Herman Wold for multivariate analysis, and its application was subsequently extended to Structural Equation Modeling (SEM) in 1975 by Wold himself (for an extensive review on PLS approach, see Esposito Vinzi, Chin, Henseler, \& Wang, 2010).

The procedure can be thought of as the analysis of two conceptually different models: 1) A structural (or inner) model that specifies the causal relationships among Latent Variables (LVs), as posited by a given theory; 2) A measurement (or outer) model that specifies the relationship of the Manifest Variables (MV) with their (hypothesized) underlying LVs - in our case, the latent constructs are the semantic (I order) and affective (II order) meanings.

The two models' equations are as follows:

$$
\begin{aligned}
& \xi_{(m, 1)}=\mathrm{B}_{(m, m)} \cdot \xi_{(m, 1)}+\tau_{(m, 1)} \\
& \mathrm{x}_{(p, 1)}=\Lambda_{(p, m)} \cdot \xi_{(m, 1)}+\tau_{(p, 1)}
\end{aligned}
$$

where the subscripts $m$ and $p$ are the number of latent and manifest variables in the model, respectively, while $\xi$ represents the LV's vector and $\mathrm{x}$ the vector of MVs. Path coefficients linking the LVs are indicated by the matrix B, while factor loadings linking MVs to LVs are represented by the matrix $\Lambda$. Finally, the $\tau$ and $\delta$ vectors indicate error terms of the structural and measurement model, respectively. The estimation algorithm is explained in the Appendix.

Wold's original PLS path modeling design does not consider higher-order LVs; each construct has to be related to a set of observed variables in order to be estimated. To address this, Lohmöller (1989) proposed a procedure for the case of hierarchical 
Table 1.

Latent Variables and selected Manifest Variables.

\begin{tabular}{ll}
\hline Latent Variables (LVs) and corresponding object & Manifest Variables (MVs) \\
\hline$\xi_{1}$ UNIVERSITY (Commitment on university) & x1 Teachers love their subjects \\
& x2 Degree of development of Italian university system \\
& x3 Degree of development of University of Salento \\
& x4 Usefulness of university studies for a job placement \\
& x5 Trust in Local Government \\
& x6 Trust in Health System \\
$\xi_{2}$ SOCIETY (Trustworthiness of social structures) & x7 Trust in Police \\
& x8 Trust in Public Administration \\
& $x 9$ Degree of development of Italy \\
& $x 10$ To see a psychologist is necessary \\
$\xi_{3}$ PSYCHO (Psychology as profession) & $x 11$ To see a psychologist is useful \\
& $x 12$ To see a psychologist is interesting \\
& $x 13$ To see a psychologist is risky \\
& $x 14$ Relying on people is difficult \\
& $x 15$ People can only rely on themselves \\
& $x 16$ The importance of understanding the world \\
$\xi_{4}$ VALUES (General values) & $x 17$ The importance of being unscrupulous \\
& $x 18$ The importance of obeying the rules \\
& $x 19$ The importance of commanding respect \\
\hline
\end{tabular}

constructs, the so-called Hierarchical Component Model or Repeated Indicators Approach, which is the most popular approach when estimating higher-order constructs through PLS (another procedure used is the Two-Step Approach; see Agarwal \& Karahanna, 2000; Diamantopoulos \& Winklhofer, 2001; Reinartz, Krafft, \& Hoyer, 2004). The procedure is very simple: The manifest indicators are used a second time to directly measure the higher-order construct; a second-order factor is directly measured by observed variables for all first-order factors.

While this approach repeats the number of MVs used, the model can be estimated using the standard PLS algorithm (Reinartz et al., 2004). The repeated indicator approach is illustrated in Figure 1.

\section{The Theoretical Model}

According to the theoretical model, the LVs $\xi_{1}$, (UNIVERSITY $^{2}$ ) $\xi_{2}$, (SOCIETY) $\xi_{3}$, (PSYCHO) $\xi_{4}$, (VALUES) were considered as independent first order constructs. This assumption of independence was made because these constructs concern separate objects (i.e. Commitment on university, Trustworthiness of the social system, Psychology as profession, General values) that lack semantic linkage between them. Consequently, in the first-order model, no causative relation ships among the constructs were assumed, which limited the analysis to a descriptive calculation of the correlation (Pearson's coefficient) among them.

The second-order construct $\left(\xi_{5}\right)$ introduced, named IMAGE, is a dimension underlying the first order LVs, and is linked (in a "reflective" way, see appendix) with all of them. In the sec-

${ }^{2}$ In the whole text caps were adopted for indicating latent constructs and italics for the corresponding objects.

${ }^{3}$ It is worth to remind that PLS path modeling lacks a well identified global optimization criterion, and then no global fitting function to assess the goodness of the model exists. The GoF index, introduced by Tenenhaus, Amato \& Esposito Vinzi (2004), represents an operational solution to this problem, being based on local measures (communality index and R-square, which are quality measures referred to each single LV) rather than on a global criterion of fitness. ond-order model, LVs at the first level hold the same absence of relationships supposed in the first-order model. Figure 2 reports the path diagram of the theoretical model, showing just the causative relationships from the second order and the first

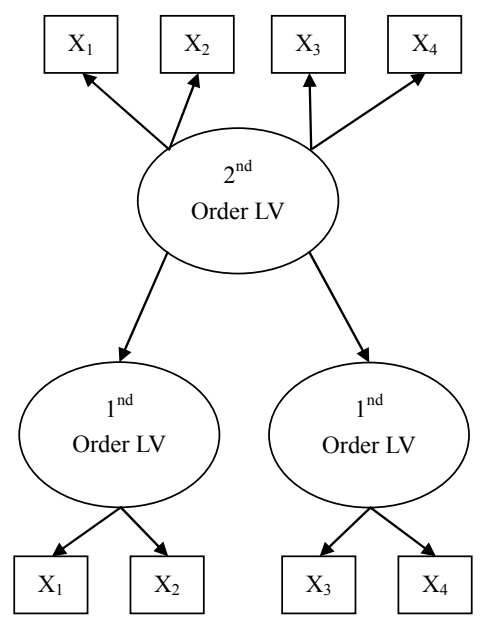

Figure 1.

PLS model building: Repeated indicators.

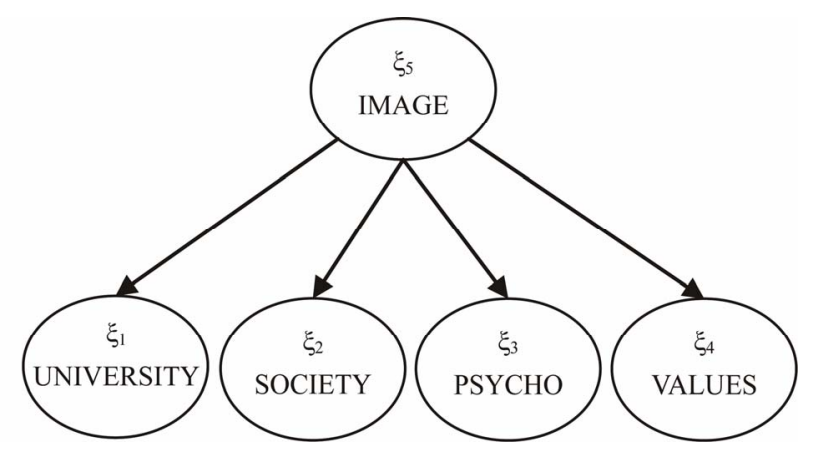

Figure 2.

Second-order model path diagram. 
order constructs, and hides the link with the manifest variables.

Composite reliability (Chin, 1998) was used to assess the internal consistency of each block of MVs (it should be $>.70$ ). Regarding the inner model, the coefficient of determination (R-square) of each dependent LV gives the local fit of the model. Goodness of Fit $(\mathrm{GoF})$ was instead taken as a goodness of fit index of the overall model ${ }^{3}$. Furthermore, for assessing the significance of path coefficients, $t$-values have been computed by bootstrapping (200 samples; $t$-values $>1.96$ significant at the .01 level).

\section{Results}

First-order model. Table 2 shows the coefficients of correlation among the four first order constructs. As one can see, 3 out of 6 correlations are statistically significant: SOCIETY-UNIVERSITY $(r=.48)$, VALUES-PSYCHO $(r=.14)$ and SOCIETYVALUES $(r=-.12)$.

Second-order model. Table 3 shows the model's quality measures. Two first order constructs (UNIVERSITY and SOCIETY) are higher than the .70 threshold; the other two (VALUES and PSYCHO) are borderline. The second order construct reaches a level (.44) far from the standard unidimensionality. SOCIETY and UNIVERSITY show the highest level of local fitness (respectively, .75 and .58). Figure 3 reports the estimated values, with the respective significance, of the path coefficients (linking second to first-order LVs). All four path

Table 2.

Correlation among first-order LVs.

\begin{tabular}{ccccc}
\hline & University & Society & Psycho & Values \\
\hline University & 1 & - & - & - \\
Society & $.48^{*}$ & 1 & - & - \\
Psycho & .02 & -.04 & 1 & - \\
Values & -.08 & $-.12^{*}$ & $.14^{*}$ & 1 \\
\hline
\end{tabular}

Table 3.

Reliability measures and Goodness of Fit.

\begin{tabular}{cccc}
\hline & Composite Reliability & R Square & GoF \\
\hline University & .726 & .575 & .37 \\
Society & .780 & .752 & \\
Values & .668 & .198 & \\
Psycho & .620 & .138 & \\
Image & .442 & - & \\
\hline
\end{tabular}

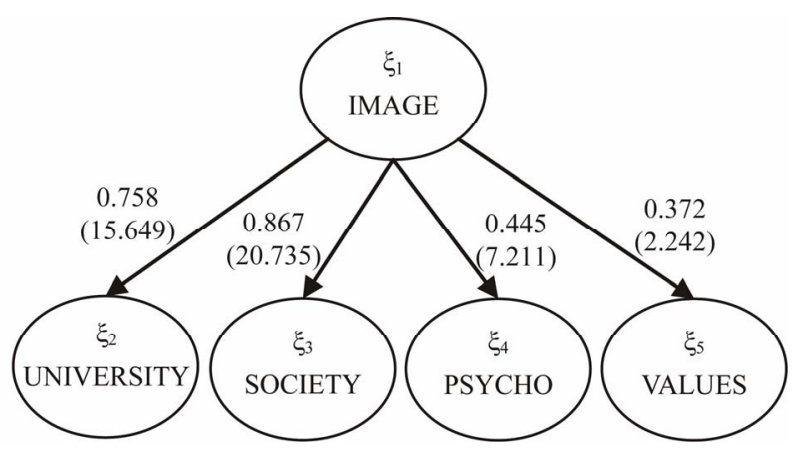

Figure 3.

Estimated path coefficients and $t$-statistics. coefficients are statistically significant; three of them-IMAGE $\rightarrow$ SOCIETY $(.87, t=20.74)$ and IMAGE $\rightarrow$ UNIVERSITY $(.76, t=15.65)$ and IMAGE $\rightarrow$ VALUES $(.45, t=7.21)$ are highly significant.

\section{Discussion}

The empirical test has provided evidence that supports the theoretical model proposed. Regarding the theoretical model and its empirical test, three main points are worth highlighting. First, the inner model, assuming causative unidirectional linkage from the second order factor to the first order factors, showed all defined linkages as being statistically significant. Moreover, these causative linkages had good indexes of fit; and the same was true for the model as a whole $(\mathrm{GoF}=.37$, $\mathrm{cfr}$. Table 3). Second, to interpret the second order construct, one has to consider that the outer model addressed the full set of items measured as part of it. This means that the second factor was defined as a construct pertaining to the meaning of the experience (as all items measured this kind of content), yet was independent from its specific semantic content (as it is the set of items taken as a whole that measured the construct, regardless of the fact that items pertained to different semantic domains/objects-i.e., Commitment to the university, Trustworthiness of the social systems, Psychology as profession, General values). In accord with our hypothesis, this way of measuring the second order factor legitimized our conceptualization of it as affective meaning - namely, the global connotation of the whole field of experience was not dependent on/constrained by the semantic specificity of the discrete objects in which the field was articulated.

Thus, taken together, the inner and outer models provided evidence supporting our hypothesis that affective meaning has a downward, causal, regulative influence on semantic meaning. In other words, the model showed how the representation of discrete objects depends on the affective connotation of the general field of experience. Incidentally, this conclusion allows us to understand the data emerging from our analysis that are otherwise counterintuitive: the associations among first order constructs (SOCIETY-UNIVERSITY, VALUES-PSYCHO, and SOCIETY-VALUES). If one conceives of sense-making in a unidimensional way-namely, only as a matter of semanticcognitive processes - these associations are hard to understand given that there are no semantic similarities/connections among the involved objects (commitment to the university, trustworthiness of the social system, general values, psychology as science). On the contrary, they can be interpreted as indirectly reflecting the effect of the affective meaning's regulative function. In other words, what the first order constructs have in common is not their semantic content, but their regulation by the affective super-ordinate meaning.

Third, it is worth noting that two out of four first order constructs (VALUE and PSYCHO) did not reach a fully satisfying level of reliability (cfr. Table 3). Furthermore, the second order construct's level of reliability was even lower. The borderline level of reliability of VALUE and PSYCHO can be understood if one considers that a second analysis on a dataset produced for other studies was carried out. However, the value of internal consistency of the second order construct would ideally be evaluated taking into account the heterogeneous content of the set of manifest variables used to measure it. Regardless, this study is not concerned with the method of measuring the con- 
structs, but rather with the linkages among them. According to that focus, the very fact that the measurement of the latent constructs was not fully efficient made it even more notable that statistically significant causative linkages emerged from the analyses.

Fourth, the strength of the causal linkages between second order and first order constructs linking each single object to the generalized super-ordinate meaning varied across the four objects. Two path coefficients (related to UNIVERSITY and SOCIETY) were high, however, one was quite high (VALUE), while another was closer to the threshold of statistical significance. This leads us to conclude that each object is only partly affected by affective meaning, thereby preserving specificity and a degree of independence for that object.

In sum, our findings showed that sense-making unfolds on two different levels, which encompass both specific meanings related to the issues addressed by the intervention (semantic meanings) and hypergeneralized meanings (i.e. affective meanings) concerning the whole experience of the individuals, which play a super-ordinate role on the former. How may these findings be used for designing and implementing cultural-based interventions in community settings according to an ecological perspective? Its is argued that our model can serve as a tool for the diagnostic phase of the intervention and has relevant implications for the actions to be planned and undertaken, as well as for general methodology. In the authors' view, two main implications can be highlighted.

First, this model leads to a new interpretation of the notion of local knowledge/local culture. If one takes into account the role of affect in shaping the experience that people make of their immediate environment, one also has to consider that when local knowledge (i.e. local culture) is elicited and communities are involved in the whole process of the intervention, there are two "horns" of culture to deal with: one horn is situatedness, the other is globality. Thus, on the one hand, one is inclined to consider the situated experience of individuals and groups and to circumscribe this experience to specific objects-mainly those directly addressed by the intervention. On the other hand, one is inclined to consider a super-ordinate level of experience that concerns the relationship between the individuals and the context at a more general level. These two levels are intertwined, so that what people perceive, think, and imagine about the specific objects (e.g. university, psychology, etc.) on which the intervention is focused, is not separable from the hypergeneralized meanings emerging from their unreflective global experience of the immediate environment.

An example of what the recognition of the affective meaning could mean at the level of intervention is provided by the actions implemented in the psychology undergraduate course our study addressed. To facilitate the transition of freshmen from high school to university and therefore increase their learning capabilities, an ad hoc setting was created: Freshmen were invited to meet each others in groups, for a planned period of time and a number of sessions (15 participants each group, lasting 2 hours, encountering 5 times). Group meetings were planned and directed in accordance with the ecological, cultural-oriented, general tenet stating that enabling actors to analyze and make sense of their local system of activity is an efficacious lever of empowerment. Accordingly, the groups' aim was to promote and support the freshmen's sense-making of their new educational role (e.g., in terms of role demands, "rules of the game", ends, goals, tools and resources character- izing the educational micro-system). As a consequence of the recognition of the affective dimension of sense-making, these groups were committed to an additional function/aim: enabling the freshmen to recognize the affective meanings crossing their shared culture, thereby regulating and constraining their individual, as well as intersubjective, ways of understanding and acting within the new educational system. Thus, in the group setting, the elaboration of being-an-undergraduate-student-ofpsychology was not limited to the cognitive task addressing the semantic objects sustaining the experience of being a student (e.g., the educational task, the learning standards, the logistic, didactic, and organizational resources and tools, the contents and structure of the programs, the implicit and explicit social norms active in the context, the attitude of the professors, and so forth). Rather, it was expanded to include the affective meanings that - due to their hypergeneralized extension-go far beyond the semantic and functional boundaries of the educational setting, encompassing the infinite (present, past, and future) elements of the freshmen's life that (directly or indirectly) are associated with the experience of being an undergraduate student (e.g., the community of peers, the family, the social environmental, the job market, and the like). In last analysis, so defined, the groups worked in a reflective setting where freshmen could analyze the rooting and the shaping of their new educational experience within/by their individual and societal identity (for details on this model of setting, its way of functioning and impact, see Venuleo \& Guidi, 2010; see also Kullasepp, 2010). To say this another way, the recognition of the salience of the affective meaning produced a shift in the psychological intervention on the educational setting; this shift was from the analysis of the identity of role to the analysis of the role of the identity.

Second, our model suggests that the actions to be implemented vary according to the autonomy of the object-i.e., the degree of independence of the cognitive-semantic representation from the hypergeneralized meaning connoting the global field of experience. The greater the autonomy, the more the action will need to focus on the object (e.g., technical training), leaving aside the general experience of the broader context (e.g., the education system, or the cultural frames). Conversely, the lower the autonomy, the more the action will have to rely upon the experience and the reflexivity of participants (e.g., groupbased self-reflexive training). This leads us to argue for the utility of introducing a diagnostic phase aimed at estimating the degree of autonomy of the object(s) in the planning of ecological culture-based intervention. For instance, take the freshmen of our study and image two scenarios of intervention: Scenario A is the one which was referred to above-namely, the one that aims to elaborate the transition into the new educational setting and the empowerment of the capability of learning. The intervention was carried out based on a diagnosis according to which the object addressed (the commitment to the university) showed a fair degree of autonomy, and such a diagnosis suggested the activation of a reflective setting.

Suppose instead that the diagnosis showed that another object, for instance English as an instrumental language, was endowed with autonomy. In this (virtual) scenario B, the intervention has a more specific aim: to favor the use of English as the main language in the educational setting (provided, of course, the setting is not an English speaking country). Consequently, while in Scenario A, the intervention required the activation of a reflective setting, the intervention in Scenario B would re- 
quire a technical-training setting focused on the specific target object - namely the diffusion of linguistic competences.

In conclusion, though the authors are aware that the soundness of the model requires further examination through repeated tests on a greater variety of datasets, objects, and settings, they feel that this study represents a first step toward a deeper comprehension of the individual-context relationship, and enriches the ecological perspective. Indeed, taking the role of affects into account can help psychologists and practitioners develop a more sophisticated sensitivity to the context, and drive them toward a more subtle calibration of culture-based interventions.

\section{REFERENCES}

Agarwal, R., \& Karahanna, E. (2000). Time flies when you're having fun: Cognitive absorption and beliefs about information technology usage. MIS Quarterly, 24, 665-694. doi:10.2307/3250951

Bronfenbrenner, U. (1979). The ecology of human development: Experiments by nature and design. Cambridge: Harvard University Press.

Bucci, W. (1997). Psychoanalysis and cognitive science. Roma: Fioriti.

Cabell, K., \& Valsiner, J. (in Press). Affective hypergeneralization: Learning from psychoanalysis. In S. Salvatore, \& T. Zittoun (Eds.), Cultural psychology and psychoanalysis. Pathways to synthesis. Charlotte, NC: Info Age Publishing.

Chin, W.W. (1998). The partial least squares approach to structural equation modeling. In G. A. Marcoulides (Ed.), Modern methods for business research (pp. 295-336). Mahwah, NJ: Lawrence Erlbaum Associates.

Carli, R., \& Salvatore, S. (2001). The image of psychology: Research on Latial population. Roma: Kappa.

Ciavolino, E., \& Al-Nasser, A. D. (2009). Comparing generalized maximum entropy and partial least squares methods for structural equation models. Journal of Nonparametric Statistics, 21, 10171036. doi:10.1080/10485250903009037

Diamantopoulos, A., \& Winklhofer, H. M. (2001). Index construction with formative indicators: An alternative to scale development. Journal of Marketing Research, 38, 269-277. doi:10.1509/jmkr.38.2.269.18845

Esposito Vinzi, V., Chin, W. W., Henseler, J., \& Wang, H. (2010). Handbook of partial least squares: Concepts, methods and applications. New York: Springer Handbooks of Computational Statistics. doi:10.1007/978-3-540-32827-8

Felner, R. D., \& Adam, A. M. (1988). The school transitional environment project. In R. H. Price, E. L. Cowen, R. P. Lorion, J. RamosMcKay, \& B. Hitchins (Eds.), Fourteen ounces of prevention: A casebook of exemplary primary prevention programs (pp. 111-122). Washington, DC: American Psychological Association.

Felner, R. D., Favazza, A., Shim, M., Brand, S., Gu, K., \& Noonan, N. (2001). Whole school improvement and restructuring as prevention and promotion. Lessons form STEP and the Project on High Perfomance Learning Communities. Journal of School Psychology, 39, 177-202. doi:10.1016/S0022-4405(01)00057-7

Freud, S. (1964). New introductory lectures on psycho-analysis. In J. Strachey (Ed.), The standard edition of the complete psychological works of Sigmund Freud (Vol. 22, pp. 1-182). London: The Hogarth Press and the Institute of Psycho-Analysis.

Guidi, M., \& Salvatore, S. (in Press). Parents' images of their children's school system. In A. Iannaccone, K. Komatsu, \& P. Marsico (Eds.), Crossing Boundaries. Intercontextual dynamics between family and school. Charlotte, NC: Information Age Publication. doi:10.1086/456198

Hawe, P., Shiell, A., \& Riley, T. (2009). Theorising interventions as events in systems. American Journal of Community Psychology, 43, 267-276. doi:10.1007/s10464-009-9229-9

Hirsch, G. B., Levine, R., \& Miller, R. L. (2007). Using system dynamics modeling to understand the impact of social change initiatives. American Journal of Community Psychology, 39, 239-243. doi:10.1007/s10464-007-9114-3

Kelly, J. (1966). Ecological constraints on mental health services. American Psychologist, 21, 535-539. doi:10.1037/h0023598

Kelly, J. (1987). An ecological paradigm: Defining mental health consultation as a preventive service. In J. Kelly, \& R. Hess (Eds.), The ecology of prevention: Illustrating mental health consultation (pp. 1-35). New York: Haworth Press.

Kelly, J. (2006). On becoming ecological: An expedition into community psychology. New York: Oxford University Press. doi:10.1093/acprof:oso/9780195173796.001.0001

Kullasepp, K. (2010). Why become a "shrink"? Psychology studies as an extension of the Self. In S. Salvatore, J. Valsiner, J. Travers Simon, \& A. Gennaro (Eds.), Yearbook of Idiographic Science (Vol. 3, pp. 95-115). Rome: Firera Publishing Group.

Lohmöller, J.B. (1989). Latent variable path modeling with partial least squares. Heidelberg: Physica-Verlag.

Matte Blanco, I. (1975). The unconscious as infinite sets. London: Duckworth.

Murphy, S. T., \& Zajonc, R. B. (1993). Affect, cognition, and awareness: Affective priming with optimal and suboptimal stimulus exposures. Journal of Personality \& Social Psychology, 64, 723-739. doi:10.1037/0022-3514.64.5.723

Osgood, C. E., Suci, G. J., \& Tannenbaum, P. H. (1957). The measurement of meaning. Chicago: University of Illinois Press.

Reinartz, W., Krafft, M., \& Hoyer, W. D. (2004). The customer relationship management process: Its measurement and impact on performance. Journal of Marketing Research, 41, 293-305. doi:10.1509/jmkr.41.3.293.35991

Salgado, J., \& Clegg, J. W. (2011). Dialogism and the psyche: Bakhtin and contemporary psychology. Culture Psychology, 17, 421-440. doi: $10.1177 / 1354067 X 11418545$

Salvatore, S., Mannarini, T., \& Rubino, F. (2004). The culture of Italian school teachers. An empirical study. School Psychology, 7, 31-70.

Salvatore, S. Mossi, P. G., \& Cazzetta, M. (2007). The satisfaction of students' families in the schools of Apulia. In Ufficio Scolastico Regionale per la Puglia (Ed.), The schools of Apulia in the backlight (pp. 333-354). Bari: Arti Grafiche Flavia.

Salvatore, S., \& Freda, M. F. (2011). Affect, unconscious and sensemaking. A psychodynamic semiotic and dialogic model. New Ideas in Psychology, 29, 119-135.

doi:10.1016/j.newideapsych.2010.06.001

Salvatore, S., \& Zittoun, T. (2010), Cultural psychology and psychoanalysis in dialogue: Issues for constructive theoretical and tethodological synergies. Roma: Firera Group Publishing.

Schensul, J. J. (2009). Community, culture and sustainability in multilevel dynamic systems intervention science. American Journal of Community Psychology, 43, 241-245. doi:10.1007/s10464-009-9228-X

Tenenhaus, M., Amato, S., \& Esposito Vinzi, V. (2004). A global goodness-of-fit index for PLS structural equation modelling. Proceedings of the XLII SIS Scientific Meeting (pp. 739-742). Padova: CLEUP.

Trickett, E. J. (2009). Multilevel community-based culturally situated interventions and community impact: An ecological perspective. American Journal of Community Psychology, 43, 257-366. doi:10.1007/s10464-009-9227-y

Turvey, M. T., Fertig, J., \& Kravetz, S.(1969). Connotative classification and proactive interference in short-term memory. Psychonomic Science, 16, 223-24.

Valsiner, J., \& Rosa J. (Eds), The Cambridge handbook of sociocultural psychology. Cambridge: Cambridge University Press.

Venuleo, C., Mossi, P., \& Salvatore, S. (Submitted). Educational subcultures and dropping out at higher education: A case study. Sociology of Education.

Venuleo, C., \& Guidi, M. (2010). The "reflexive training setting" as a model for working on the meanings that shape student's view of their role. A case study on psychology freshmen. In S. Salvatore, J. Valsiner, J. Travers Simon, \& A. Gennaro (Eds.), Yearbook of Idiographic Science (Vol. 3, pp. 65-94). Rome: Firera Publishing Group.

Wold, H. (1966). Estimation of principal components and related models by iterative least squares. In P. R. Krishnajah (Ed.), Multivariate 
analysis (pp. 391-420). NewYork: Academic Press.

Wold, H. (1975). Path models with latent variables: The NIPALS ap- proach. In H. M. Blalock (Ed.), Quantitative sociology (pp. 307-357). New York: Seminar Press.

\section{Appendix}

The PLS algorithm.

The parameters estimation (Ciavolino \& Al-Nasser, 2009) is based on a double approximation of the $\operatorname{LVs} \xi_{j}$ (with $j=1, \cdots$, $m$ ). The external estimation $\mathrm{y}_{j}$, obtained as the product of the block of $\mathrm{MVs}_{j}$ (considered as the matrix units for variables) and the outer weights $\mathrm{w}_{j}$ (which represent the estimation of measurement coefficients, $\Lambda$ ). The internal estimation $\mathrm{z}_{j}$, obtained as the product of the external estimation of $\xi_{j}, \mathrm{y}_{j}$, and the inner weights $e_{j}$.

According with the relationship among MVs and LVs hypothesized, outer weights are computed as:

$$
\mathrm{w}_{j}=\mathrm{X}_{j}^{\prime} \cdot \mathrm{z}_{j}
$$

for Mode A (reflective relationship), and:

$$
\mathrm{w}_{j}=\left(\mathrm{X}_{j}^{\prime} \mathrm{X}_{j}\right)^{-1} \mathrm{X}_{j}^{\prime} \cdot \mathrm{z}_{j}
$$

for Mode B (formative relationship).

The inner weights $\mathrm{e}_{j, i}$, in the centroid scheme, are defined as the sign of the correlation between the connected estimated $\mathrm{y}_{j}$ and $\mathrm{y}_{i}$, with $i \neq j$.

The PLS algorithm starts by initializing outer weights to one for the first MV of each LV; then, the parameters estimation is performed, until the convergence, by iteratively computing:

1) External estimation, $y_{j}=X_{j} \cdot z_{j}$;

2) Internal estimation, $\mathrm{z}_{j}=\sum_{j \neq i} e_{j, i} \cdot \mathrm{y}_{i}$;

3) Outer weights estimation, with Mode A or B.

The causal paths among LVs (the coefficients in the B matrix) are obtained through Ordinary Least Squares (OLS) method. 